\title{
Atom-Economic Synthesis of 4-Pyrones from Diynones and Water
}

\author{
Yan-Li Xu ${ }^{1, \dagger}{ }^{,}$Qing-Hu Teng ${ }^{2,+}$, Wei Tong ${ }^{2}$, Heng-Shan Wang ${ }^{2}$, Ying-Ming Pan ${ }^{2, *}$ and \\ Xian-Li Ma ${ }^{1, *}$ \\ 1 College of Pharmacy, Guilin Medical University, Guilin 541004, China; yanlixu@163.com \\ 2 State Key Laboratory for Chemistry and Molecular Engineering of Medicinal Resources, \\ School of Chemistry and Pharmaceutical Sciences of Guangxi Normal University, Guilin 541004, China; \\ qinghuteng@163.com (Q.-H.T.); m18778391736@163.com (W.T.); whengshan@163.com (H.-S.W.) \\ * Correspondence: panym2013@hotmail.com (Y.-M.P.); mxl78@glmc.edu.cn (X.-L.M.); \\ Tel.: +86-773-584-6279 (Y.-M.P.); Fax: +86-773-5803930 (Y.-M.P.); +86-773-5893619 (X.-L.M.) \\ + These authors contributed equally to this work. \\ Academic Editor: Georgiy B. Shul'pin \\ Received: 21 December 2016; Accepted: 5 January 2017; Published: 10 January 2017
}

\begin{abstract}
Transition-metal-free synthesis of 4-pyrones via TfOH-promoted nucleophilic addition/ cyclization of diynones and water has been developed. This transformation is simple, atom economical and environmentally benign, providing rapid and efficient access to substituted 4-pyrones.
\end{abstract}

Keywords: 4-pyrones; diynones; water; transition-metal-free

\section{Introduction}

Water $\left(\mathrm{H}_{2} \mathrm{O}\right)$ is inexpensive, safe, and environmentally friendly [1]. It is the most economic and eco-friendly solvent available in Nature and therefore highly desirable for chemical reactions [2]. Generally, water offers several "green chemistry" benefits as a solvent in organic transformations, including high efficiency, lower cost, ease of process, green and environmental protection $[3,4]$. Recently, there are many reports of clean transformations in water medium [5-19], such as coupling reactions [20-30], cyclizations [31-34], Michael additions [35-39], and condensations [40,41]. Additionally, $\mathrm{H}_{2} \mathrm{O}$ also participates in organic reactions as a nucleophile $[42,43]$ to provide various kinds of functional compounds such as imidazo[1,2-a]pyridines [44], amino acid salts [45], $\alpha$-amino ketones [46], and 1,3-oxazinan-2-ones [47]. Thus, the studies of organic reactions in aqueous solvents or $\mathrm{H}_{2} \mathrm{O}$-participating reactions are attractive in synthetic chemistry.

4-Pyrones are heterocycles with multiple biological activities [48-50], which are widely found in biologically active natural products and functional chemicals [51-59]. Particularly, phenoxans, funicones and rapicones possess potent anti-HIV activity (Figure 1) [60-62]. In general, 4-pyrones are prepared via the well-known condensation cyclization reaction of carbonyl compounds with polystep reactions [63-67]. Additionally, a transformation of isoxazoles to substituted pyran-4-ones in the presence of $\mathrm{Mo}(\mathrm{CO})_{6}$ and $\mathrm{HCO}_{2} \mathrm{H}$ in a two-step procedure was established [68]. Although these reported methods have made significant contributions to the applications of 4-pyrones in pharmacology and food manufacture [69], the development of efficient and practical synthetic methods for 4-pyrones from easily accessible starting materials is still highly desirable. Continuing our interest in the conversion of alkynes to heterocycles [70-77], herein, we would like to describe an efficient, transition-metal-free synthesis of 4-pyrones through $\mathrm{TfOH}$-promoted cyclization of diynones. Water acts as both the substrate and solvent, obviating the need for an organic co-solvent. Overall, the reaction is atom-economical and environmentally benign. 

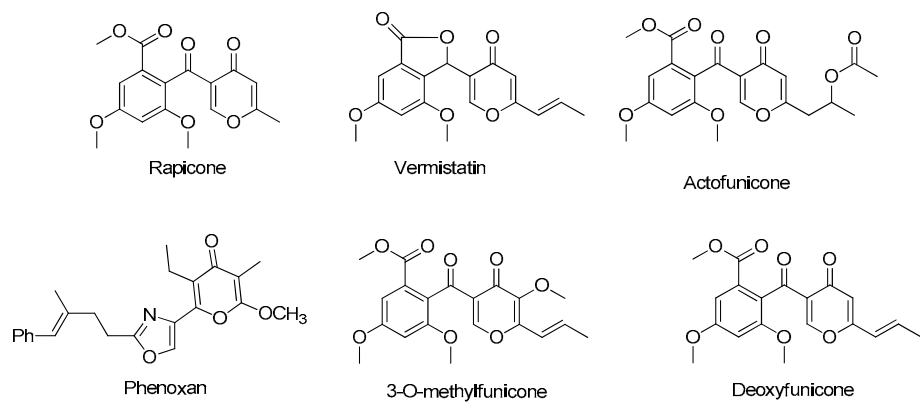

Figure 1. 4-Pyrones disclosed as biologically active organic molecules.

\section{Results and Discussion}

1,5-Diphenylpenta-1,4-diyn-3-one (1a) was chosen as model substrate to identify the optimal conditions for this reaction (Table 1). Originally, the reaction was carried out in the presence of 1 equiv. $\mathrm{TfOH}$ for $24 \mathrm{~h}$ to afford the desired product $2 \mathrm{a}$ in $70 \%$ yield (Table 1, entry 1 ). When other acid catalysts such as $\mathrm{CH}_{3} \mathrm{COOH}, \mathrm{PTSA}, \mathrm{HCl}, \mathrm{H}_{3} \mathrm{PO}_{4}$ and $\mathrm{PhCOOH}$ were screened, the yield of 2a decreased (Table 1 , entries 2-6). Further experiments demonstrated that decreasing the amount of $\mathrm{TfOH}$ was detrimental to the yield of $\mathbf{2 a}$ (Table 1, entries 7 and 8 ), and no obvious improvement of yield was noted by using 2 equiv. of TfOH (Table 1, entry 9). Poor yield of $2 \mathbf{a}$ was obtained when the reaction was performed at $80{ }^{\circ} \mathrm{C}$, while not much change was noted between $100{ }^{\circ} \mathrm{C}$ and $130{ }^{\circ} \mathrm{C}$ (Table 1 , entries 10 and 11). In addition, an $83 \%$ yield was achieved when the reaction time was extended to $36 \mathrm{~h}$ (Table 1, entry 12 ). Thus, the best conditions for this transformation involved 1 equiv. of $\mathrm{TfOH}$ in $\mathrm{H}_{2} \mathrm{O}$ at $100{ }^{\circ} \mathrm{C}$ for $36 \mathrm{~h}$.

Table 1. Optimization of reaction conditions ${ }^{a}$.

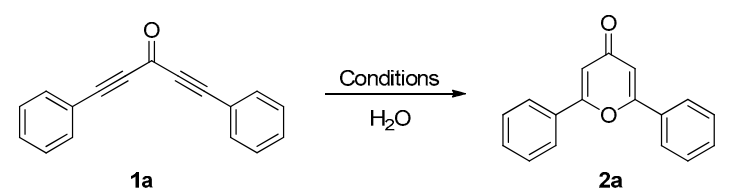

\begin{tabular}{cccc}
\hline Entry & Catalyst & Time (h) & Yield (\%) $^{\mathbf{b}}$ \\
\hline 1 & TfOH & 24 & 70 \\
2 & $\mathrm{CH}_{3} \mathrm{COOH}$ & 24 & 0 \\
3 & $\mathrm{PTSA}$ & 24 & 50 \\
4 & $\mathrm{HCl}$ & 24 & 0 \\
5 & $\mathrm{H}_{3} \mathrm{PO}$ & 24 & 0 \\
6 & $\mathrm{PhCOHH}_{4}$ & 24 & 10 \\
$7^{\mathrm{c}}$ & $\mathrm{TfOH}$ & 24 & 10 \\
$8^{\mathrm{d}}$ & $\mathrm{TfOH}$ & 24 & 50 \\
$9^{\mathrm{e}}$ & $\mathrm{TfOH}$ & 24 & 80 \\
$1^{\mathrm{f}}$ & $\mathrm{TfOH}$ & 24 & 20 \\
$11^{\mathrm{g}}$ & $\mathrm{TfOH}$ & 24 & 75 \\
$1^{2}$ & $\mathrm{TfOH}$ & 36 & 83
\end{tabular}

a Reaction conditions: 1a $(0.5 \mathrm{mmol})$, catalyst (1 equiv.), $\mathrm{H}_{2} \mathrm{O}(1 \mathrm{~mL})$, at $100{ }^{\circ} \mathrm{C} ;{ }^{\mathrm{b}}$ Isolated yields; ${ }^{\mathrm{c}} \mathrm{TfOH}$ (0.2 equiv.); ${ }^{\mathrm{d}} \mathrm{TfOH}\left(0.5\right.$ equiv.); ${ }^{\mathrm{e}} \mathrm{TfOH}\left(2\right.$ equiv.); ${ }^{\mathrm{f}}$ At $80{ }^{\circ} \mathrm{C} ; \mathrm{g}$ The reaction was carried out in a sealed tube at $130^{\circ} \mathrm{C}$.

Under the optimized reaction conditions, the one-pot reaction worked well using all kinds of diynones, as shown in Scheme 1. Firstly, various symmetric diynones were identified as suitable substrates for the reaction and provided the desired products in moderate to good yields (Scheme 1, $\mathbf{2 b} \mathbf{- 2 j}$ ). Aryl groups with electron-donating groups (EDG) gave satisfactory yields (Scheme 1, 2b-2d and $\mathbf{2 f - 2 h}$ ), whereas aryl groups with electron-withdrawing groups (EWG) afforded slightly lower yields (Scheme 1, 2e). Gratifyingly, aliphatic diynones worked smoothly to generate the corresponding 
cyclization products $\mathbf{2 i}$ and $\mathbf{2} \mathbf{j}$ in $50 \%$ and $57 \%$, respectively (Scheme 1, $\mathbf{2} \mathbf{i}$ and $\mathbf{2 j}$ ). After exploring the reaction substrate scope of symmetric diynones, we next examined asymmetric diynones substrates. To our delight, the corresponding 4-pyrones products were obtained in moderate to good yields under the standard conditions (Scheme 1, 2k-2r). The desired products $\mathbf{2 k}-\mathbf{2 q}$ were obtained in $55 \%-78 \%$ yields when asymmetric diynones substrates $\mathbf{1 k}-\mathbf{1 q}\left(\mathrm{R}^{2}=\mathrm{Ph}, \mathrm{R}^{1}=\right.$ aryl- or alkyl-) were subjected to this reaction. Obviously, aryl groups with electron-donating groups gave higher yields than diynones featuring electron-withdrawing groups on the phenyl ring (Scheme 1, 2l and $\mathbf{2 m}$ vs. $2 \mathbf{n}$ and $\mathbf{2 p}$ ). Notably, diynone $\mathbf{1 p}$, which possess an electron-withdrawing group at the ortho-position of the phenyl ring $\left(\mathrm{R}^{1}=2-\mathrm{Cl}-\mathrm{Ph}, \mathrm{R}^{2}=\mathrm{Ph}\right)$ reacted readily to afford $2 \mathbf{p}$ in $61 \%$ yield (Scheme $\left.1,2 p\right)$. Furthermore, diynone 1q, which bear both a EDG-incorporated aryl ring and a EWG-incorporated aryl ring $\left(\mathrm{R}^{1}=4\right.$-OMe-Ph, $\mathrm{R}^{2}=4$-F-Ph) also participated well in the reaction and offered $2 \mathrm{q}$ in $63 \%$ yield (Scheme 1, 2q). Finally, diynone $1 \mathbf{r}$ also worked smoothly to give $2 \mathbf{r}$ in $50 \%$ yield (Scheme 1, 2r).

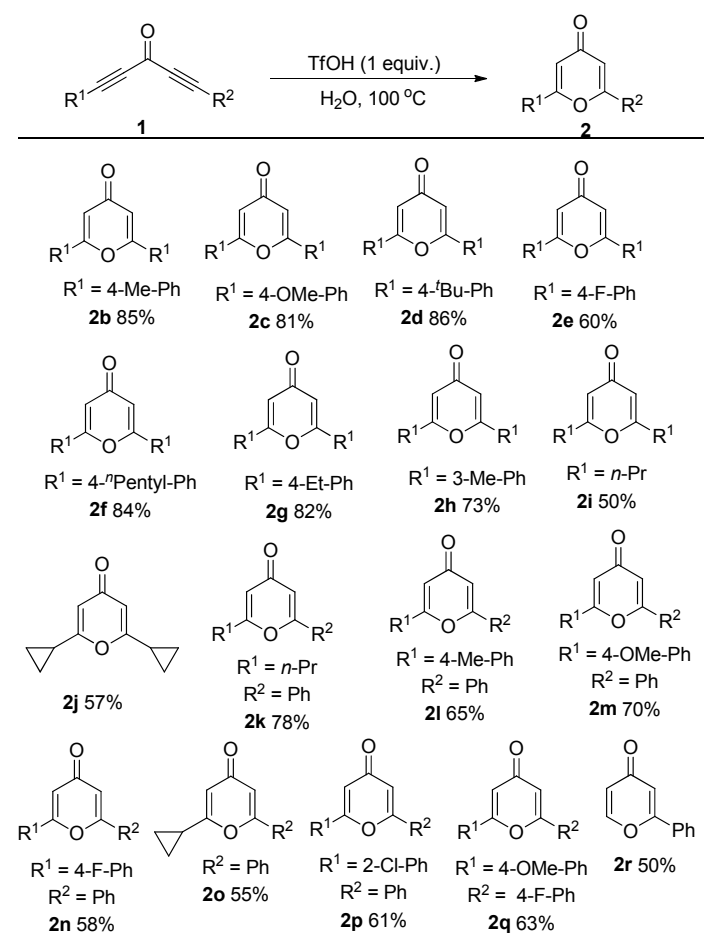

Scheme 1. Synthesis of 4-pyrone derivatives a,b ${ }^{a}$ Reaction conditions: $\mathbf{1}$ ( $\left.0.5 \mathrm{mmol}\right), \mathrm{TfOH}$ (1 equiv.), $\mathrm{H}_{2} \mathrm{O}(1 \mathrm{~mL})$, at $100{ }^{\circ} \mathrm{C}, 36 \mathrm{~h} ;{ }^{\mathrm{b}}$ Isolated yields.

To better understand the reaction mechanism, we carried out control experiments as outlined in Scheme 2. Deuterium-labeled $\mathrm{D}_{2} \mathrm{O}$ was used in the reaction with diynone 1a to give the deuterium-labeled product $2 \mathbf{a}-\mathbf{d}$ in $80 \%$ yield, where over $95 \%$ of deuterium was incorporated into the cyclization product.

This result demonstrated that $\mathrm{H}_{2} \mathrm{O}$ was introduced into the 4-pyrones. Moreover, an $\mathrm{O}^{18}$-labeled experiment further showed that $\mathrm{H}_{2} \mathrm{O}$ reacted with diynones to form 4-pyrones.

On the basis of the above results and existing literature [78], a plausible mechanistic description of the nucleophilic addition and cyclization reaction is shown in Scheme 3. First, the carbonyl of the diynone substrate was activated by $\mathrm{TfOH}$, followed by nucleophilic addition of $\mathrm{H}_{2} \mathrm{O}$ to the carbon-carbon triple bond of diynone and keto-enol tautomerization $[79,80]$ to form intermediate $\mathbf{A}$. Then intermediate $\mathbf{A}$ was converted to $\mathbf{B}$ through protonation and $\mathbf{C}-\mathbf{C}$ bond rotation, which was promoted by elevated temperature. Subsequently, an intramolecular nucleophilic attack of the oxhydryl group to the carbon-carbon triple bond of $\mathbf{B}$ lead to a cyclization intermediate $\mathbf{C}$. Finally, deprotonation of $\mathbf{C}$ gave the desired 4-pyrone $\mathbf{2}$. 
<smiles>O=C(C#Cc1ccccc1)C#Cc1ccccc1</smiles>

$1 a$<smiles>O=C(C#Cc1ccccc1)C#Cc1ccccc1</smiles>

$1 a$<smiles>[2H]c1c(-c2ccccc2)oc(-c2ccccc2)c([2H])c1=O</smiles>

2a-d $80 \%$ yield (>95\%-d)<smiles>O=c1cc(-c2ccccc2)oc(-c2ccccc2)c1</smiles>

$\mathrm{O}^{18}$-2a $78 \%$ yield $\left(>90 \%-\mathrm{O}^{18}\right)$

Scheme 2. Control experiments.

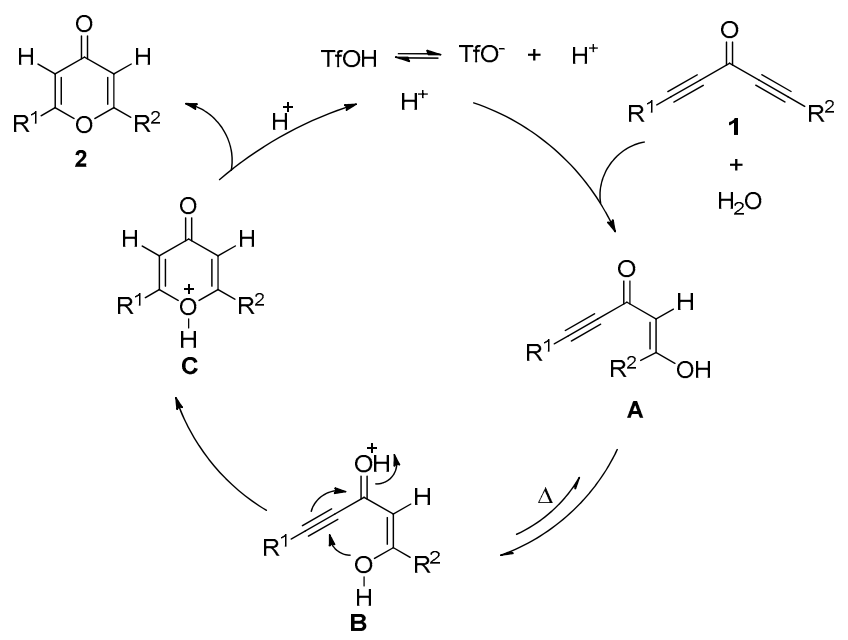

Scheme 3. Proposed mechanism.

The treatment of 1,5-diphenylpenta-1,4-diyn-3-one $1 \mathrm{a}$ in $\mathrm{H}_{2} \mathrm{O}$ at $100{ }^{\circ} \mathrm{C}$ for $36 \mathrm{~h}$ in the presence of $\mathrm{TfOH}$ afforded the corresponding cyclization product $2 \mathbf{a}$ in $83 \%$ yield. The preparation of this compound 2a on gram-scale afforded $53 \%$ of the isolated product (Scheme 4 ).<smiles>O=C(C#Cc1ccccc1)C#Cc1ccccc1</smiles>

1a<smiles>CC(O)CCCC(=O)O</smiles>

$\mathrm{H}_{2} \mathrm{O}, 100^{\circ} \mathrm{C}$<smiles>O=c1cc(-c2ccccc2)oc(-c2ccccc2)c1</smiles>

2a $83 \%(0.1029 \mathrm{~g}, 0.5 \mathrm{mmol}$ scale $)$ $53 \%(0.6572 \mathrm{~g}, 5 \mathrm{mmol}$ scale)

Scheme 4. Gram-scale synthesis.

\section{Materials and Methods}

\subsection{General Information}

All manipulations were performed under an air atmosphere unless otherwise stated. Column chromatography was performed on silica gel (300-400 mesh). NMR spectra were obtained using 
an Avance 500 spectrometer $\left({ }^{1} \mathrm{H}\right.$ at $500 \mathrm{MHz}$ and ${ }^{13} \mathrm{C}$ at $\left.125 \mathrm{MHz}\right)$ or an Avance 400 spectrometer $\left({ }^{1} \mathrm{H}\right.$ at $400 \mathrm{MHz}$ and ${ }^{13} \mathrm{C}$ at $\left.100 \mathrm{MHz}\right)$ (Bruker Corporation, Karlsruhe, Germany). IR spectra were recorded on a Nicolet ESP 360 FT-IR spectrometer (Nicolet, Madison, WI, USA) and only major peaks are reported in $\mathrm{cm}^{-1}$. High resolution mass spectra (HRMS) were recorded on an Exactive Mass Spectrometer (Thermo Fisher Scientific, Waltham, MA, USA) equipped with ESI or APCI ionization sources. Unless stated otherwise, commercial reagents were used without further purification. All reagents were weighed and handled at room temperature. Compounds $1 \mathbf{a}-\mathbf{1 r}$ were prepared by the reported methods $[78,81]$. The NMR spectra and HRMS spectra of the products can be found in the Supplementary Materials.

\subsection{General Procedure for the Synthesis of Compound 2}

The reaction mixture of $\mathbf{1}(0.5 \mathrm{mmol})$, $\mathrm{TfOH}$ (1 equiv.) and $\mathrm{H}_{2} \mathrm{O}(1 \mathrm{~mL})$ in a $15 \mathrm{~mL}$ test tube was stirred at $100^{\circ} \mathrm{C}$ for $36 \mathrm{~h}$, and monitored periodically by TLC. Upon completion, the reaction mixture was diluted with water $(5 \mathrm{~mL})$ and extracted with ethyl acetate $(3 \times 5 \mathrm{~mL})$. The combined organic layers were washed with water and brine, dried over $\mathrm{MgSO}_{4}$ and filtered. The solvent was removed under vacuum. The residue was purified by flash column chromatography (petroleum ether and ethyl acetate, $v / v=5: 1$ to 2:1) to afford 4-pyrones 2 (Scheme 5).

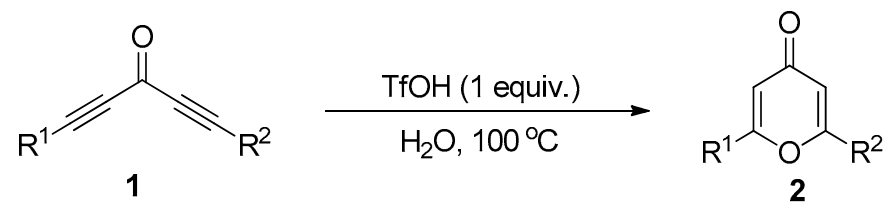

Scheme 5. Synthesis of Compounds 2.

2,6-Diphenyl-4H-pyran-4-one (2a) [82]. The general procedure was used with 1,5-diphenylpenta1,4-diyn-3-one ( $115.04 \mathrm{mg}, 0.5 \mathrm{mmol}, 1$ equiv.) and water $(1 \mathrm{~mL})$. The crude obtained was purified by column chromatography (petroleum ether/ethyl acetate $=5: 1$ to $2: 1$ ) to afford the product as a yellow solid (102.90 mg, 83\%); m.p. 135.3-136.2 ${ }^{\circ} \mathrm{C}$ (lit: $\left.139-140{ }^{\circ} \mathrm{C}\right) ;{ }^{1} \mathrm{H}-\mathrm{NMR}\left(500 \mathrm{MHz}, \mathrm{CDCl}_{3}\right) \delta 7.89-7.82$ $(\mathrm{m}, 4 \mathrm{H}), 7.55-7.50(\mathrm{~m}, 6 \mathrm{H}), 6.81(\mathrm{~s}, 2 \mathrm{H}) \mathrm{ppm} ;{ }^{13} \mathrm{C}-\mathrm{NMR}\left(125 \mathrm{MHz}, \mathrm{CDCl}_{3}\right) \delta 180.2,163.3,131.4,131.4$, 129.1, 125.9, 111.4 ppm; IR (KBr): 3060, 2925, 1647, 1614, 1604, 1493, 1450, 1392, 943, 770, $683 \mathrm{~cm}^{-1}$; HRMS $(m / z)$ (APCI): calcd. for $\mathrm{C}_{17} \mathrm{H}_{13} \mathrm{O}_{2} 249.0917$ [M + $\mathrm{H}^{+}$]; found 249.0906 .

2,6-Di-p-tolyl-4H-pyran-4-one (2b) [78]. The general procedure was used with 1,5-di-p-tolylpenta1,4-diyn-3-one (129.05 mg, $0.5 \mathrm{mmol}, 1$ equiv.) and water $(1 \mathrm{~mL})$. The crude obtained was purified by column chromatography (petroleum ether/ethyl acetate $=5: 1$ to $2: 1$ ) to afford the product as a yellow solid (117.35 mg, 85\%); m.p. $180.5-183.1{ }^{\circ} \mathrm{C}$ (lit: $178{ }^{\circ} \mathrm{C}$ ); ${ }^{1} \mathrm{H}-\mathrm{NMR}\left(500 \mathrm{MHz}, \mathrm{CDCl}_{3}\right) \delta 7.74$ $(\mathrm{d}, J=8.2 \mathrm{~Hz}, 42 \mathrm{H}), 7.32(\mathrm{~d}, J=8.0 \mathrm{~Hz}, 4 \mathrm{H}), 6.76(\mathrm{~s}, 2 \mathrm{H}), 2.43(\mathrm{~s}, 6 \mathrm{H}) \mathrm{ppm} ;{ }^{13} \mathrm{C}-\mathrm{NMR}\left(125 \mathrm{MHz}, \mathrm{CDCl}_{3}\right)$ $\delta$ 180.4, 163.4 141.9, 129.8, 128.7, 125.8, 110.7, 21.5 ppm; IR (KBr): 3066, 1646, 1605, 1507, 1413, 1383, 942, 819, $478 \mathrm{~cm}^{-1}$; HRMS ( $\mathrm{m} / z$ ) (APCI): calcd. for $\mathrm{C}_{19} \mathrm{H}_{17} \mathrm{O}_{2} 277.1230\left[\mathrm{M}+\mathrm{H}^{+}\right]$; found 277.1219.

2,6-Bis(4-methoxyphenyl)-4H-pyran-4-one (2c) [82]. The general procedure was used with 1,5-bis (4-methoxyphenyl)penta-1,4-diyn-3-one ( $145.05 \mathrm{mg}, 0.5 \mathrm{mmol}, 1$ equiv.) and water $(1 \mathrm{~mL})$. The crude obtained was purified by column chromatography (petroleum ether / ethyl acetate $=5: 1$ to 2:1) to afford the product as a yellow solid (124.78 mg, 81\%); m.p. $190-193.8^{\circ} \mathrm{C}$ (lit: $\left.189-191{ }^{\circ} \mathrm{C}\right) ;{ }^{1} \mathrm{H}-\mathrm{NMR}(500 \mathrm{MHz}$, $\left.\mathrm{CDCl}_{3}\right) \delta 7.79(\mathrm{~d}, J=8.9 \mathrm{~Hz}, 4 \mathrm{H}), 7.02(\mathrm{~d}, J=8.9 \mathrm{~Hz}, 4 \mathrm{H}), 6.70(\mathrm{~s}, 2 \mathrm{H}), 3.88(\mathrm{~s}, 6 \mathrm{H}) \mathrm{ppm} ;{ }^{13} \mathrm{C}-\mathrm{NMR}$ $\left(125 \mathrm{MHz}, \mathrm{CDCl}_{3}\right) \delta 163.2,162.1,134.4,127.5,123.9,114.5,109.7,55.5$ ppm; IR (KBr): 2983, 2875, 2765, $1651,1607,1507,1387,1262,1226,1177,1020,829 \mathrm{~cm}^{-1}$; HRMS ( $\left.m / z\right)$ (APCI): calcd. for $\mathrm{C}_{19} \mathrm{H}_{17} \mathrm{O}_{4}$ $309.1129\left[\mathrm{M}+\mathrm{H}^{+}\right]$; found 309.1115 .

2,6-Bis(4-(tert-butyl)phenyl)-4H-pyran-4-one (2d) [82]. The general procedure was used with 1,5-bis(4(tert-butyl)phenyl)penta-1,4-diyn-3-one ( $171.10 \mathrm{mg}, 0.5 \mathrm{mmol}, 1$ equiv.) and water ( $1 \mathrm{~mL})$. The crude 
obtained was purified by column chromatography (petroleum ether/ethyl acetate $=5: 1$ to $2: 1$ ) to afford the product as a yellow solid (154.89 mg, 86\%); m.p. $192.5-193.1{ }^{\circ} \mathrm{C}$ (lit: $\left.192-194{ }^{\circ} \mathrm{C}\right) ;{ }^{1} \mathrm{H}-\mathrm{NMR}$ $\left(500 \mathrm{MHz}, \mathrm{CDCl}_{3}\right) \delta 7.79(\mathrm{~d}, J=7.2 \mathrm{~Hz}, 4 \mathrm{H}), 7.54(\mathrm{~d}, J=7.4 \mathrm{~Hz}, 4 \mathrm{H}), 6.81(\mathrm{~s}, 2 \mathrm{H}), 1.36(\mathrm{~s}, 18 \mathrm{H}) \mathrm{ppm}$; ${ }^{13} \mathrm{C}-\mathrm{NMR}\left(125 \mathrm{MHz}, \mathrm{CDCl}_{3}\right) \delta 180.5,163.5,155.0,128.6,126.0,125.7,34.9,31.0$ ppm; IR (KBr): 3064, 3003, 2998, 2970, 2868, 1715, 1667, 1650, 1450, 1340, 1250, $910 \mathrm{~cm}^{-1}$; HRMS ( $\mathrm{m} / \mathrm{z}$ ) (APCI): calcd. for $\mathrm{C}_{25} \mathrm{H}_{29} \mathrm{O}_{2} 361.2169\left[\mathrm{M}+\mathrm{H}^{+}\right.$]; found 361.2153.

2,6-Bis(4-fluorophenyl)-4H-pyran-4-one (2e) [82]. The general procedure was used with 1,5-bis (4-fluorophenyl)penta-1,4-diyn-3-one ( $133.12 \mathrm{mg}, 0.5 \mathrm{mmol}, 1$ equiv.) and water ( $1 \mathrm{~mL})$. The crude obtained was purified by column chromatography (petroleum ether/ethyl acetate $=5: 1$ to $2: 1$ ) to afford the product as a white solid $(85.22 \mathrm{mg}, 60 \%)$; m.p. $160-161.3{ }^{\circ} \mathrm{C}$ (lit: $\left.167-170{ }^{\circ} \mathrm{C}\right) ;{ }^{1} \mathrm{H}-\mathrm{NMR}$ $\left(500 \mathrm{MHz}, \mathrm{CDCl}_{3}\right) \delta 7.84(\mathrm{dd}, J=8.5,5.2 \mathrm{~Hz}, 4 \mathrm{H}), 7.22(\mathrm{t}, J=8.4 \mathrm{~Hz}, 4 \mathrm{H}), 6.75(\mathrm{~s}, 2 \mathrm{H}) \mathrm{ppm} ;{ }^{13} \mathrm{C}-\mathrm{NMR}$ $\left(125 \mathrm{MHz}, \mathrm{CDCl}_{3}\right) \delta 179.9,164.6(\mathrm{~d}, J=253.4 \mathrm{~Hz}), 162.5,128.1(\mathrm{~d}, J=8.8 \mathrm{~Hz}), 127.6(\mathrm{~d}, J=3.3 \mathrm{~Hz})$, 116.5(d, J = 22.2 Hz), 111.3 ppm; IR (KBr): 3059, 2924, 1662, 1599, 1504, 1417, 1380, 1241, 1223, 1160, $837 \mathrm{~cm}^{-1}$; HRMS ( $/ \mathrm{z} / \mathrm{z}$ ) (APCI): calcd. for $\mathrm{C}_{17} \mathrm{H}_{11} \mathrm{~F}_{2} \mathrm{O}_{2} 285.0729\left[\mathrm{M}+\mathrm{H}^{+}\right]$; found 285.0716.

2,6-Bis(4-pentylphenyl)-4H-pyran-4-one (2f). The general procedure was used with 1,5-bis(4-pentylphenyl) penta-1,4-diyn-3-one (185.11 mg, $0.5 \mathrm{mmol}, 1$ equiv.) and water $(1 \mathrm{~mL})$. The crude obtained was purified by column chromatography (petroleum ether/ethyl acetate $=5: 1$ to $2: 1$ ) to afford the product as a yellow solid $(163.06 \mathrm{mg}, 84 \%) ; \mathrm{m} . p .66 .7-67.9{ }^{\circ} \mathrm{C} ;{ }^{1} \mathrm{H}-\mathrm{NMR}\left(500 \mathrm{MHz}, \mathrm{CDCl}_{3}\right) \delta 7.76(\mathrm{~d}, J=8.1 \mathrm{~Hz}$, $4 \mathrm{H}), 7.32(\mathrm{~d}, J=8.1 \mathrm{~Hz}, 4 \mathrm{H}), 6.77(\mathrm{~s}, 2 \mathrm{H}), 2.70-2.66(\mathrm{~m}, 4 \mathrm{H}), 1.69-1.61(\mathrm{~m}, 4 \mathrm{H}), 1.36-1.33(\mathrm{~m}, 8 \mathrm{H}), 0.90$ $(\mathrm{t}, J=6.9 \mathrm{~Hz}, 6 \mathrm{H}) \mathrm{ppm} ;{ }^{13} \mathrm{C}-\mathrm{NMR}\left(125 \mathrm{MHz}, \mathrm{CDCl}_{3}\right) \delta 180.5,163.5,146.9,129.2,128.9,125.9,110.7,35.8$, 31.4, 30.8, 22.5, 13.9 ppm; IR (KBr): 3032, 2956, 2929, 2857, 1717, 1649, 1609, 1419, 1380, 1186, 944, 849, $649 \mathrm{~cm}^{-1}$; HRMS ( $\left.m / z\right)$ (APCI): calcd. for $\mathrm{C}_{27} \mathrm{H}_{33} \mathrm{O}_{2} 389.2482\left[\mathrm{M}+\mathrm{H}^{+}\right]$; found 389.2466.

2,6-Bis(4-ethylphenyl)-4H-pyran-4-one (2g) [78]. The general procedure was used with 1,5-bis(4-ethylphenyl) penta-1,4-diyn-3-one (143.07 mg, $0.5 \mathrm{mmol}, 1$ equiv.) and water $(1 \mathrm{~mL})$. The crude obtained was purified by column chromatography (petroleum ether/ethyl acetate $=5: 1$ to 2:1) to afford the product as a brown solid $(124.70 \mathrm{mg}, 82 \%)$; m.p. $119.5-121.5^{\circ} \mathrm{C} ;{ }^{1} \mathrm{H}-\mathrm{NMR}\left(500 \mathrm{MHz}, \mathrm{CDCl}_{3}\right) \delta 7.77(\mathrm{~d}, J=8.1 \mathrm{~Hz}$, $4 \mathrm{H}), 7.34(\mathrm{~d}, J=8.1 \mathrm{~Hz}, 4 \mathrm{H}), 6.77(\mathrm{~s}, 2 \mathrm{H}), 2.72(\mathrm{q}, J=7.6 \mathrm{~Hz}, 4 \mathrm{H}), 1.28(\mathrm{t}, J=7.6 \mathrm{~Hz}, 6 \mathrm{H}) \mathrm{ppm} ;{ }^{13} \mathrm{C}-\mathrm{NMR}$ $\left(125 \mathrm{MHz}, \mathrm{CDCl}_{3}\right) \delta 180.4,163.5,148.2,128.9,128.6,125.9,110.6,28.8,15.2$ ppm; IR (KBr): 3070, 2965, $2875,1647,1610,1510,1451,1420,1383,1187,1014,945,837,643 \mathrm{~cm}^{-1}$; HRMS ( $m / z$ ) (APCI): calcd. for $\mathrm{C}_{21} \mathrm{H}_{21} \mathrm{O}_{2} 305.1543\left[\mathrm{M}+\mathrm{H}^{+}\right]$; found 305.1532.

2,6-Di-m-tolyl-4H-pyran-4-one (2h) [78]. The general procedure was used with 1,5-di-m-tolylpenta1,4-diyn-3-one (129.05 mg, $0.5 \mathrm{mmol}, 1$ equiv.) and water $(1 \mathrm{~mL})$. The crude obtained was purified by column chromatography (petroleum ether/ethyl acetate $=5: 1$ to $2: 1$ ) to afford the product as a light yellow solid (100.78 mg, 73\%); m.p. 73.5-75.5 ${ }^{\circ} \mathrm{C} ;{ }^{1} \mathrm{H}-\mathrm{NMR}\left(500 \mathrm{MHz}, \mathrm{CDCl}_{3}\right) \delta 7.66-7.62(\mathrm{t}, J=7.6 \mathrm{~Hz}$, $4 \mathrm{H}), 7.41(\mathrm{t}, J=7.6 \mathrm{~Hz}, 2 \mathrm{H}), 7.34(\mathrm{~d}, J=7.6 \mathrm{~Hz}, 2 \mathrm{H}), 6.78(\mathrm{~s}, 2 \mathrm{H}), 2.45(\mathrm{~s}, 6 \mathrm{H}) \mathrm{ppm} ;{ }^{13} \mathrm{C}-\mathrm{NMR}(125 \mathrm{MHz}$, $\left.\mathrm{CDCl}_{3}\right) \delta 163.6,138.9,132.1,131.4,129.0,126.5,123.1,111.3,21.5$ ppm; IR (KBr): 3063, 2923, 1646, 1611, $1485,1384,1260,1075,929,784,694,435 \mathrm{~cm}^{-1}$; HRMS ( $m / z$ ) (APCI): calcd. for $\mathrm{C}_{19} \mathrm{H}_{17} \mathrm{O}_{2} 277.1230$ $\left[\mathrm{M}+\mathrm{H}^{+}\right]$; found 277.1219.

2,6-Dipropyl-4H-pyran-4-one (2i) [78]. The general procedure was used with undeca-4,7-diyn-6-one ( $81.05 \mathrm{mg}, 0.5 \mathrm{mmol}, 1$ equiv.) and water $(1 \mathrm{~mL})$. The crude obtained was purified by column chromatography (petroleum ether/ethyl acetate $=5: 1$ to 2:1) to afford the product as a brown oil (45.03 mg, 50\%); ${ }^{1} \mathrm{H}-\mathrm{NMR}\left(500 \mathrm{MHz}, \mathrm{CDCl}_{3}\right) \delta 6.05(\mathrm{~s}, 1 \mathrm{H}), 2.44(\mathrm{t}, J=7.5 \mathrm{~Hz}, 4 \mathrm{H}), 1.69-1.61(\mathrm{~m}, 4 \mathrm{H})$, $0.95(\mathrm{td}, J=7.4,1.1 \mathrm{~Hz}, 6 \mathrm{H}) \mathrm{ppm} ;{ }^{13} \mathrm{C}-\mathrm{NMR}\left(125 \mathrm{MHz}, \mathrm{CDCl}_{3}\right) \delta 180.6,169.1,113.0,35.4,20.1,13.3 \mathrm{ppm}$; IR (KBr): $3437,2965,2875,1663,1619,1411,1398,1148,933,864 \mathrm{~cm}^{-1}$; HRMS ( $m / z$ ) (APCI): calcd. for $\mathrm{C}_{11} \mathrm{H}_{14} \mathrm{O}_{2}$ 181.1230 [M + $\left.\mathrm{H}^{+}\right]$; found 181.1221.

2,6-Dicyclopropyl-4H-pyran-4-one (2j) [78]. The general procedure was used with 1,5-dicyclopropylpenta1,4-diyn-3-one (79.04 mg, $0.5 \mathrm{mmol}, 1$ equiv.) and water $(1 \mathrm{~mL})$. The crude obtained was purified by column chromatography (petroleum ether/ethyl acetate $=5: 1$ to 2:1) to afford the product as a white 
solid (50.19 mg, 57\%); m.p. 146.7-150.7 ${ }^{\circ} \mathrm{C} ;{ }^{1} \mathrm{H}-\mathrm{NMR}\left(500 \mathrm{MHz}, \mathrm{CDCl}_{3}\right) \delta 6.04(\mathrm{~s}, 2 \mathrm{H}), 1.72(\mathrm{tt}, J=8.3$, $5.0 \mathrm{~Hz}, 2 \mathrm{H}), 1.00-0.95(\mathrm{~m}, 4 \mathrm{H}), 0.92-0.88(\mathrm{~m}, 4 \mathrm{H}) \mathrm{ppm} ;{ }^{13} \mathrm{C}-\mathrm{NMR}\left(125 \mathrm{MHz}, \mathrm{CDCl}_{3}\right) \delta 179.5,168.6$, 111.1, 13.7, 7.8 ppm; IR (KBr): 3045, 3010, 2955, 1655, 1602, 1586, 1401, 1095, 1053, $858 \mathrm{~cm}^{-1}$; HRMS $(m / z)$ (APCI): calcd. for $\mathrm{C}_{11} \mathrm{H}_{13} \mathrm{O}_{2}$ 177.0917 [M + $\mathrm{H}^{+}$]; found 177.0908.

2-Phenyl-6-propyl-4H-pyran-4-one (2k) [78]. The general procedure was used with 1-phenylocta1,4-diyn-3-one ( $98.04 \mathrm{mg}, 0.5 \mathrm{mmol}, 1$ equiv.) and water $(1 \mathrm{~mL})$. The crude obtained was purified by column chromatography (petroleum ether/ethyl acetate $=5: 1$ to $2: 1$ ) to afford the product as a brown solid (83.50 mg, 78\%); m.p. 49.8-51.5 ${ }^{\circ} \mathrm{C} ;{ }^{1} \mathrm{H}-\mathrm{NMR}\left(500 \mathrm{MHz}, \mathrm{CDCl}_{3}\right) \delta 7.75(\mathrm{dd}, J=7.7,1.8 \mathrm{~Hz}, 1 \mathrm{H})$, 7.51-7.46 (m, 1H), $6.72(\mathrm{~s}, 1 \mathrm{H}), 6.19(\mathrm{~s}, 1 \mathrm{H}), 2.60(\mathrm{t}, J=7.5 \mathrm{~Hz}, 1 \mathrm{H}), 1.82-1.73(\mathrm{~m}, 1 \mathrm{H}), 1.03(\mathrm{t}, J=7.4 \mathrm{~Hz}$, 1H) ppm; ${ }^{13} \mathrm{C}-\mathrm{NMR}\left(125 \mathrm{MHz}, \mathrm{CDCl}_{3}\right) \delta 180.1,168.8,163.6,131.5,131.3,129.0,125.8,114.0,111.1,35.6$, 20.3, 13.5 ppm; IR (KBr): 3060, 2926, 1653, 1617, 1493, 1450, 1409, 1061, 937, 866, 772, $691 \mathrm{~cm}^{-1}$; HRMS $(\mathrm{m} / z)$ (APCI): calcd. for $\mathrm{C}_{14} \mathrm{H}_{15} \mathrm{O}_{2} 215.1074\left[\mathrm{M}+\mathrm{H}^{+}\right.$]; found 215.1065 .

2-Phenyl-6-(p-tolyl)-4H-pyran-4-one (21) [83]. The general procedure was used with 1-phenyl-5-( $p$-tolyl) penta-1,4-diyn-3-one $(112.04 \mathrm{mg}, 0.5 \mathrm{mmol}, 1$ equiv.) and water $(1 \mathrm{~mL})$. The crude obtained was purified by column chromatography (petroleum ether/ethyl acetate $=5: 1$ to 2:1) to afford the product as a yellow solid $(85.18 \mathrm{mg}, 65 \%) ;$ m.p. $155.1-156.4{ }^{\circ} \mathrm{C}$ (lit: $\left.150{ }^{\circ} \mathrm{C}\right) ;{ }^{1} \mathrm{H}-\mathrm{NMR}\left(500 \mathrm{MHz}, \mathrm{CDCl}_{3}\right) \delta$ 7.88-7.83 (m, 2H), $7.75(\mathrm{~d}, J=8.2 \mathrm{~Hz}, 2 \mathrm{H}), 7.54-7.51(\mathrm{~m}, 3 \mathrm{H}), 7.32(\mathrm{~d}, J=8.1 \mathrm{~Hz}, 2 \mathrm{H}), 6.83-6.78(\mathrm{~m}, 2 \mathrm{H})$, $2.44(\mathrm{~s}, 3 \mathrm{H}) \mathrm{ppm} ;{ }^{13} \mathrm{C}-\mathrm{NMR}\left(125 \mathrm{MHz}, \mathrm{CDCl}_{3}\right) \delta 180.4,163.6,163.3,142.0,131.5,131.4,129.8,129.1$, 128.6, 125.91, 125.86, 111.3, 110.7, 21.5 ppm; IR (KBr): 3064, 2922, 2854, 1646, 1606, 1448, 1413, 1387, 943, $816 \mathrm{~cm}^{-1}$; HRMS ( $/ \mathrm{z}$ ) (APCI): calcd. for $\mathrm{C}_{18} \mathrm{H}_{15} \mathrm{O}_{2} 263.1074\left[\mathrm{M}+\mathrm{H}^{+}\right]$; found 263.1061 .

2-(4-Methoxyphenyl)-6-phenyl-4H-pyran-4-one (2m) [78]. The general procedure was used with 1-(4-methoxyphenyl)-5-phenylpenta-1,4-diyn-3-one (130.04 mg, $0.5 \mathrm{mmol}, 1$ equiv.) and water (1 mL). The crude obtained was purified by column chromatography (petroleum ether/ethyl acetate $=5: 1$ to 2:1) to afford the product as a brown solid $\left(97.33 \mathrm{mg}\right.$, 70\%); m.p. $161.3-162.2^{\circ} \mathrm{C}$ (lit: $162{ }^{\circ} \mathrm{C}$ ); ${ }^{1} \mathrm{H}-\mathrm{NMR}$ $\left(500 \mathrm{MHz}, \mathrm{CDCl}_{3}\right) \delta 7.82(\mathrm{dd}, J=6.6,3.0 \mathrm{~Hz}, 2 \mathrm{H}), 7.78(\mathrm{~d}, J=8.9 \mathrm{~Hz}, 2 \mathrm{H}), 7.50(\mathrm{dd}, J=5.0,1.7 \mathrm{~Hz}, 3 \mathrm{H})$, $7.00(\mathrm{~d}, J=8.9 \mathrm{~Hz}, 2 \mathrm{H}), 6.76(\mathrm{~d}, J=1.7 \mathrm{~Hz}, 1 \mathrm{H}), 6.70(\mathrm{~d}, J=1.7 \mathrm{~Hz}, 1 \mathrm{H}), 3.86(\mathrm{~s}, 3 \mathrm{H}) \mathrm{ppm} ;{ }^{13} \mathrm{C}-\mathrm{NMR}$ $\left(125 \mathrm{MHz}, \mathrm{CDCl}_{3}\right) \delta 180.2,163.3,163.0,162.2,131.5,131.2,129.0,127.5,125.8,123.6,114.5,111.1,109.8$, 55.4 ppm; IR (KBr): 3443, 3067, 2900, 2843, 1647, 1604, 1509, 1448, 1423, 1383, 1023, 832, 767, $684 \mathrm{~cm}^{-1}$; HRMS $(m / z)(A P C I)$ : calcd. for $\mathrm{C}_{18} \mathrm{H}_{15} \mathrm{O}_{3} 279.1014\left[\mathrm{M}+\mathrm{H}^{+}\right.$]; found 279.1013.

2-(4-Fluorophenyl)-6-phenyl-4H-pyran-4-one (2n). The general procedure was used with 1-(4-fluorophenyl)5-phenylpenta-1,4-diyn-3-one (124.03 mg, $0.5 \mathrm{mmol}, 1$ equiv.) and water ( $1 \mathrm{~mL})$. The crude obtained was purified by column chromatography (petroleum ether/ethyl acetate $=5: 1$ to $2: 1$ ) to afford the product as a yellow solid (77.16 mg, 58\%); m.p. $145.5-150.6{ }^{\circ} \mathrm{C} ;{ }^{1} \mathrm{H}-\mathrm{NMR}\left(500 \mathrm{MHz}, \mathrm{CDCl}_{3}\right) \delta 7.89-7.82$ $(\mathrm{m}, 4 \mathrm{H}), 7.56-7.51(\mathrm{~m}, 3 \mathrm{H}), 7.22(\mathrm{t}, J=8.5 \mathrm{~Hz}, 2 \mathrm{H}), 6.82(\mathrm{~d}, J=1.8 \mathrm{~Hz}, 1 \mathrm{H}), 6.77(\mathrm{~d}, J=1.8 \mathrm{~Hz}, 1 \mathrm{H}) \mathrm{ppm}$; ${ }^{13} \mathrm{C}-\mathrm{NMR}\left(125 \mathrm{MHz}, \mathrm{CDCl}_{3}\right) \delta 180.1,165.6,163.6,163.4,162.5,131.5,131.3,129.2,128.1$ (d, J = 8.9 Hz), 127.6, 125.9, 116.4 (d, J = 22.1 Hz), $111.3(\mathrm{~d}, J=24.2 \mathrm{~Hz}) \mathrm{ppm}$; IR (KBr): 3061, 2924, 1659, 1505, 1508, 1417, 1449, 1388, 1232, $1162 \mathrm{~cm}^{-1}$; HRMS ( $\left.m / z\right)$ (APCI): calcd. for $\mathrm{C}_{17} \mathrm{H}_{12} \mathrm{FO}_{2} 267.0823\left[\mathrm{M}+\mathrm{H}^{+}\right]$; found 267.0813 .

2-Cyclopropyl-6-phenyl-4H-pyran-4-one (2o) [84]. The general procedure was used with 1-cyclopropyl5-phenylpenta-1,4-diyn-3-one ( $97.04 \mathrm{mg}, 0.5 \mathrm{mmol}, 1$ equiv.) and water $(1 \mathrm{~mL})$. The crude obtained was purified by column chromatography (petroleum ether/ethyl acetate $=5: 1$ to 2:1) to afford the product as a yellow solid (58.33 mg, 55\%); m.p. $106.5-107.8^{\circ} \mathrm{C}$ (lit: $\left.106{ }^{\circ} \mathrm{C}\right) ;{ }^{1} \mathrm{H}-\mathrm{NMR}\left(500 \mathrm{MHz}, \mathrm{CDCl}_{3}\right) \delta 7.67$ $(\mathrm{dd}, J=7.9,1.7 \mathrm{~Hz}, 2 \mathrm{H}), 7.50-7.45(\mathrm{~m}, 3 \mathrm{H}), 6.69(\mathrm{~d}, J=2.1 \mathrm{~Hz}, 1 \mathrm{H}), 6.23(\mathrm{~d}, J=2.1 \mathrm{~Hz}, 1 \mathrm{H}), 1.90(\mathrm{tt}$, $J=7.9,5.4 \mathrm{~Hz}, 1 \mathrm{H}), 1.12(\mathrm{tt}, J=4.7,2.5 \mathrm{~Hz}, 4 \mathrm{H}) \mathrm{ppm} ;{ }^{13} \mathrm{C}-\mathrm{NMR}\left(125 \mathrm{MHz}, \mathrm{CDCl}_{3}\right) \delta 179.8,169.5,162.7$, 131.3, 131.2, 129.0, 125.6, 111.6, 111.0, 14.1, 8.5 ppm; IR (KBr): 3059, 2927, 1651, 1609, 1544, 1496, 1448, 1394, 1253, 1193, 1087, 931, 878, 766, $685 \mathrm{~cm}^{-1}$; HRMS ( $\mathrm{m} / \mathrm{z}$ ) (APCI): calcd. for $\mathrm{C}_{14} \mathrm{H}_{13} \mathrm{O}_{2} 213.0917$ $\left[\mathrm{M}+\mathrm{H}^{+}\right]$; found 213.0908 . 
2-(2-Chlorophenyl)-6-phenyl-4H-pyran-4-one (2p) [85]. The general procedure was used with 1-(2-chlorophenyl)-5-phenylpenta-1,4-diyn-3-one (132.02 mg, $0.5 \mathrm{mmol}, 1$ equiv.) and water (1 mL). The crude obtained was purified by column chromatography (petroleum ether/ethyl acetate $=5: 1$ to $2: 1$ ) to afford the product as a yellow solid (86.03 mg, 61\%); m.p. 123.5-124.6 ${ }^{\circ} \mathrm{C}$ (lit: $\left.122-124{ }^{\circ} \mathrm{C}\right)$; ${ }^{1} \mathrm{H}-\mathrm{NMR}\left(400 \mathrm{MHz}, \mathrm{CDCl}_{3}\right) \delta 7.86-7.81(\mathrm{~m}, 2 \mathrm{H}), 7.60(\mathrm{dd}, J=7.5,1.8 \mathrm{~Hz}, 1 \mathrm{H}), 7.57-7.54(\mathrm{~m}, 1 \mathrm{H})$, $7.52-7.48(\mathrm{~m}, 2 \mathrm{H}), 7.48-7.46(\mathrm{~m}, 1 \mathrm{H}), 7.44(\mathrm{dd}, J=6.6,1.7 \mathrm{~Hz}, 1 \mathrm{H}), 7.41(\mathrm{dd}, J=7.4,1.4 \mathrm{~Hz}, 1 \mathrm{H}), 6.86(\mathrm{~d}$, $J=2.2 \mathrm{~Hz}, 1 \mathrm{H}), 6.67(\mathrm{~d}, J=2.2 \mathrm{~Hz}, 1 \mathrm{H}) \mathrm{ppm} ;{ }^{13} \mathrm{C}-\mathrm{NMR}\left(100 \mathrm{MHz}, \mathrm{CDCl}_{3}\right) \delta 178.0,164.1,162.6,132.8$, $131.9,131.5,131.4,131.2,130.9,130.7,129.1,127.2,126.0,116.8,111.2$ ppm; IR (KBr): 3059, 2931, 1667, 1650, 1600, 1580, 1403, 1250, 1000, 910, $665 \mathrm{~cm}^{-1}$; HRMS (m/z) (ESI): calcd. for $\mathrm{C}_{17} \mathrm{H}_{12} \mathrm{ClO}_{2} 283.0528$ $\left[\mathrm{M}+\mathrm{H}^{+}\right]$; found 283.0513 .

2-(4-Fluorophenyl)-6-(4-methoxyphenyl)-4H-pyran-4-one (2q) [85]. The general procedure was used with 1-(4-fluorophenyl)-5-(4-methoxyphenyl)penta-1,4-diyn-3-one (139.04 mg, $0.5 \mathrm{mmol}, 1$ equiv.) and water $(1 \mathrm{~mL})$. The crude obtained was purified by column chromatography (petroleum ether/ethyl acetate $=5: 1$ to $2: 1)$ to afford the product as a yellow solid $(93.27 \mathrm{mg}, 63 \%) ;$ m.p. $138.7-140.5{ }^{\circ} \mathrm{C}$ (lit: $\left.144-148^{\circ} \mathrm{C}\right) ;{ }^{1} \mathrm{H}-\mathrm{NMR}\left(400 \mathrm{MHz}, \mathrm{CDCl}_{3}\right) \delta$ 7.86-7.82 (m, 2H), 7.80-7.77 (m, 2H), 7.27-7.18 (m, 2H), 7.05-7.00 (m, 2H), $6.72(\mathrm{dd}, J=3.7,1.9 \mathrm{~Hz}, 2 \mathrm{H}), 3.89$ (s, 3H) ppm; ${ }^{13} \mathrm{C}-\mathrm{NMR}\left(100 \mathrm{MHz}, \mathrm{CDCl}_{3}\right) \delta 180.2$, 165.8, 163.4, 163.3, $162.2(\mathrm{~d}, J=8.9 \mathrm{~Hz}), 128.1(\mathrm{~d}, J=8.8 \mathrm{~Hz}), 127.8(\mathrm{~d}, J=3.3 \mathrm{~Hz}), 127.6,123.6,116.4$ $(\mathrm{d}, J=22.1 \mathrm{~Hz}), 114.6,111.0,109.9,55.5$ ppm; IR (KBr): 3673, 3067, 2969, 1657, 1610, 1509, 1422, 1385 , 1270, 1227, 1169, 1074, 1021, $841 \mathrm{~cm}^{-1}$; HRMS (m/z) (ESI): calcd. for $\mathrm{C}_{18} \mathrm{H}_{14} \mathrm{FO}_{3} 297.0929\left[\mathrm{M}+\mathrm{H}^{+}\right]$; found 297.0913 .

2-Phenyl-4H-pyran-4-one (2r) [86]. The general procedure was used with 1-phenylpenta-1,4-diyn-3-one (77.02 $\mathrm{mg}, 0.5 \mathrm{mmol}, 1$ equiv.) and water $(1 \mathrm{~mL})$. The crude obtained was purified by column chromatography (petroleum ether/ethyl acetate $=5: 1$ to $2: 1$ ) to afford the product as a yellow solid (43.02 mg, 50\%); yellow solid; m.p. $102.2-103.5{ }^{\circ} \mathrm{C}$ (lit: $\left.100-102{ }^{\circ} \mathrm{C}\right) ;{ }^{1} \mathrm{H}-\mathrm{NMR}\left(400 \mathrm{MHz}, \mathrm{CDCl}_{3}\right) \delta 7.84$ $(\mathrm{d}, J=5.8 \mathrm{~Hz}, 1 \mathrm{H}), 7.74(\mathrm{dd}, J=7.9,1.7 \mathrm{~Hz}, 2 \mathrm{H}), 7.51-7.44(\mathrm{~m}, 3 \mathrm{H}), 6.78(\mathrm{~d}, J=2.3 \mathrm{~Hz}, 1 \mathrm{H}), 6.38(\mathrm{dd}$, $J=5.8,2.3 \mathrm{~Hz}, 1 \mathrm{H}) \mathrm{ppm} ;{ }^{13} \mathrm{C}-\mathrm{NMR}\left(100 \mathrm{MHz}, \mathrm{CDCl}_{3}\right) \delta$ 179.0, 163.9, 154.8, 131.4, 131.0, 129.0, 125.7, 117.0, 112.3 ppm; IR (KBr): 3090, 1675, 1650, 1590, 1549, 1490, 1450, 1402, 1350, 1050, 931, 875, 795, 730, $650 \mathrm{~cm}^{-1} ; \operatorname{HRMS}(\mathrm{m} / z)(\mathrm{ESI})$ : calcd. for $\mathrm{C}_{11} \mathrm{H}_{9} \mathrm{O}_{2} 173.0604\left[\mathrm{M}+\mathrm{H}^{+}\right]$; found 173.0603 .

\subsection{Control Experiments}

\subsubsection{Deuterium Labeling Experiments}

The reaction mixture of $\mathbf{1}(0.5 \mathrm{mmol}), \mathrm{TfOH}$ (1 equiv.), and $\mathrm{D}_{2} \mathrm{O}(1 \mathrm{~mL})$ in a $15 \mathrm{~mL}$ test tube was stirred at $100{ }^{\circ} \mathrm{C}$ for $36 \mathrm{~h}$, and monitored periodically by TLC. Upon completion, the reaction mixture was diluted with water $(5 \mathrm{~mL})$ and extracted with ethyl acetate $(3 \times 5 \mathrm{~mL})$. The combined organic layers were washed with water and brine, dried over $\mathrm{MgSO}_{4}$ and filtered. The solvent was removed under vacuum. The residue was purified by flash column chromatography (petroleum ether and ethyl acetate, $v / v=5: 1$ to 2:1) to afford 4-pyrone $\mathbf{2 a - d}(100.04 \mathrm{mg}, 80 \%)$ as a yellow solid; m.p. $116.1-119.5^{\circ} \mathrm{C}$; ${ }^{1} \mathrm{H}-\mathrm{NMR}\left(500 \mathrm{MHz}, \mathrm{CDCl}_{3}\right) \delta$ 7.90-7.83 (m, 4H), 7.55-7.51 (m, 6H), $6.84(\mathrm{~s}, 0.12 \mathrm{H})$ (Scheme 6).

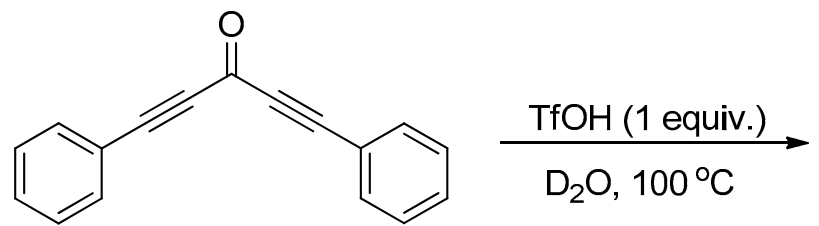

$1 a$<smiles>[2H]c1c(-c2ccccc2)oc(-c2ccccc2)c([2H])c1=O</smiles>

2a-d $80 \%$ yield (>95\%-d)

Scheme 6. Deuterium Labeling Experiments. 


\subsection{2. $\mathrm{O}^{18}$-Labelling Experiment}

The reaction mixture of $\mathbf{1 a}(0.5 \mathrm{mmol}), \mathrm{TfOH}$ (1 equiv.), and $\mathrm{H}_{2} \mathrm{O}^{18}(1 \mathrm{~mL})$ in a $15 \mathrm{~mL}$ test tube was stirred at $100{ }^{\circ} \mathrm{C}$ for $36 \mathrm{~h}$, and monitored periodically by TLC. Upon completion, the reaction mixture was diluted with water $(5 \mathrm{~mL})$ and extracted with ethyl acetate $(3 \times 5 \mathrm{~mL})$. The combined organic layers were washed with water and brine, dried over $\mathrm{MgSO}_{4}$ and filtered. The solvent was removed under vacuum. The residue was purified by flash column chromatography (petroleum ether and ethyl acetate, $v / v=5: 1$ to 2:1) to afford 4-pyrone $\mathbf{O}^{18} \mathbf{- 2 a}(78 \%)$ (Scheme 7).<smiles>O=C(C#Cc1ccccc1)C#Cc1ccccc1</smiles>

$1 a$

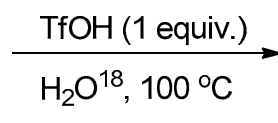

$$
\text { O" }
$$

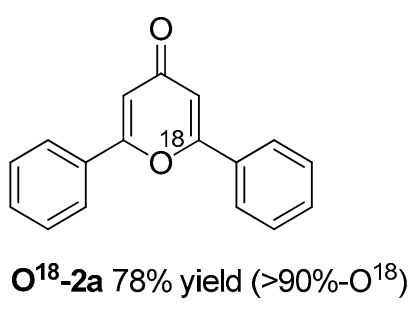

\subsubsection{Gram-Scale Synthesis}

The reaction mixture of $\mathbf{1 a}(5 \mathrm{mmol}), \mathrm{TfOH}\left(1\right.$ equiv.) and $\mathrm{H}_{2} \mathrm{O}(10 \mathrm{~mL})$ in a $50 \mathrm{~mL}$ round-bottom flask was stirred at $100{ }^{\circ} \mathrm{C}$ for $36 \mathrm{~h}$, and monitored periodically by TLC. Upon completion, the reaction mixture was diluted with water $(30 \mathrm{~mL})$ and extracted with ethyl acetate $(3 \times 30 \mathrm{~mL})$. The combined organic layers were washed with water and brine, dried over $\mathrm{MgSO}_{4}$ and filtered. The solvent was removed under vacuum. The residue was purified by flash column chromatography (petroleum ether and ethyl acetate, $v / v=5: 1$ to 2:1) to afford 4-pyrone 2a (53\%) (Scheme 8).<smiles>O=C(C#Cc1ccccc1)C#Cc1ccccc1</smiles>

1a<smiles>O=c1cc(-c2ccccc2)oc(-c2ccccc2)c1</smiles>

2a

$53 \%(0.6572 \mathrm{~g}, 5 \mathrm{mmol}$ scale $)$

Scheme 8. Gram-Scale Synthesis.

\section{Conclusions}

We have developed a simple and efficient transition-metal-free method for the synthesis of substituted 4-pyrones from diynones and $\mathrm{H}_{2} \mathrm{O}$. Water is a cheap, green and readily available staring material, which converted to the desired 4-pyrone products via a nucleophilic addition/cyclization/ dehydrogenation process. The operational simplicity, good yields, and environmentally benign nature of this method make it an attractive route to 4-pyrones. Further studies on the applications of 4-pyrones in drug design are currently ongoing in our laboratory.

Supplementary Materials: Supplementary materials can be accessed at: http://www.mdpi.com/1420-3049/22/ 1/109/s1: copies of NMR spectra and HRMS spectra of products.

Acknowledgments: We would like to thank the National Natural Science Foundation of China (21362002 and 81260472), Guangxi Natural Science Foundation of China (2014GXNSFDA118007 and 2016GXNSFEA380001), Project of Guangxi Department of Education (KY2014037), State Key Laboratory for Chemistry and Molecular Engineering of Medicinal Resources (CMEMR2014-A02 and CMEMR2012-A20), the Fund of Guangxi Key Laboratory of Functional Phytochemicals Research and Utilization (FPRU2015-2), and Guangxi's Medicine Talented Persons Small Highland Foundation (1306). 
Author Contributions: Yan-Li Xu and Qing-Hu Teng conceived and designed the experiments. The experimental work was conducted by Qing-Hu Teng under the supervision of Ying-Ming Pan and Xian-Li Ma who are the lead author; Qing-Hu Teng and Wei Tong analyzed the data; Heng-Shan Wang contributed reagents/materials/analysis tools; Yan-Li Xu and Qing-Hu Teng wrote the paper.

Conflicts of Interest: The authors declare no conflict of interest.

\section{Abbreviations}

$\begin{array}{ll}\text { TfOH } & \text { trifluoromethanesulfonic acid } \\ \text { PTSA } & \text { 4-methylbenzenesulfonic acid } \\ \mathrm{HCl} & \text { hydrochloric acid } \\ \mathrm{HIV} & \text { human immunodeficiency virus } \\ \mathrm{HOAc} & \text { acetic acid } \\ \mathrm{Ph} & \text { phenyl } \\ \mathrm{Me} & \text { methyl } \\ \mathrm{OMe} & \text { methoxyl } \\ \mathrm{Et} & \text { ethyl } \\ { }^{t} \mathrm{Bu} & \text { tertiary butyl } \\ { }^{n} \mathrm{Pr} & \text { n-propyl } \\ \mathrm{NMR} & \text { nuclear magnetic resonance } \\ \mathrm{HRMS} & \text { high-resolution mass }\end{array}$

\section{References}

1. Padiya, K.J.; Gavade, S.; Kardile, B.; Tiwari, M.; Bajare, S.; Mane, M.; Gaware, V.; Varghese, S.; Harel, D.; Kurhade, S. Unprecedented “In Water" Imidazole Carbonylation: Paradigm Shift for Preparation of Urea and Carbamate. Org. Lett. 2012, 14, 2814-2817. [CrossRef] [PubMed]

2. Narayan, S.; Muldoon, J.; Finn, M.G.; Fokin, V.V.; Kolb, H.C.; Sharpless, K.B. “On Water”: Unique Reactivity of Organic Compounds in Aqueous Suspension. Angew. Chem. Int. Ed. 2005, 44, 3275-3279. [CrossRef] [PubMed]

3. Pirrung, M.C.; Sarma, K.D. Multicomponent Reactions Are Accelerated in Water. J. Am. Chem. Soc. 2004, 126, 444-445. [CrossRef] [PubMed]

4. DeSimone, J.M. Practical Approaches to Green Solvents. Science 2002, 297, 799-803. [CrossRef] [PubMed]

5. Powner, M.W.; Zheng, S.-L.; Szostak, J.W. Multicomponent Assembly of Proposed DNA Precursors in Water. J. Am. Chem. Soc. 2012, 134, 13889-13895. [CrossRef] [PubMed]

6. Lindstedt, E.; Ghosh, R.; Olofsson, B. Metal-Free Synthesis of Aryl Ethers in Water. Org. Lett. 2013, 15, 6070-6073. [CrossRef] [PubMed]

7. Li, C.J. Organic Reactions in Aqueous Media-With a Focus on Carbon-Carbon Bond Formation. Chem. Rev. 1999, 93, 2023-2035. [CrossRef]

8. Huang, Y.-T.; Lu, S.-Y.; Yi, C.-L.; Lee, C.-F. Iron-Catalyzed Synthesis of Thioesters from Thiols and Aldehydes in Water. J. Org. Chem. 2014, 79, 4561-4568. [CrossRef] [PubMed]

9. Hao, X.; Xu, Z.M.; Lu, H.F.; Dai, X.D.; Yang, T.; Lin, X.C.; Ren, F. Mild and Regioselective N-Alkylation of 2-Pyridones in Water. Org. Lett. 2015, 17, 3382-3385. [CrossRef]

10. Sberegaeva, A.V.; Zavalij, P.Y.; Vedernikov, A.N. Oxidation of a Monomethylpalladium(II) Complex with $\mathrm{O}_{2}$ in Water: Tuning Reaction Selectivity to Form Ethane, Methanol, or Methylhydroperoxide. J. Am. Chem. Soc. 2016, 138, 1446-1455. [CrossRef] [PubMed]

11. Egami, H.; Katsuki, T. Fe(salan)-Catalyzed Asymmetric Oxidation of Sulfides with Hydrogen Peroxide in Water. J. Am. Chem. Soc. 2007, 129, 8940-8941. [CrossRef] [PubMed]

12. Shaikh, T.M.; Emmanuvel, A.L.; Sudalai, A. NaIO 4 -Mediated Selective Oxidation of Alkylarenes and Benzylic Bromides/Alcohols to Carbonyl Derivatives Using Water as Solvent. J. Org. Chem. 2006, 71, 5043-5046. [CrossRef] [PubMed]

13. Liu, X.S.; Wang, Z.T.; Cheng, X.M.; Li, C.Z. Silver-Catalyzed Decarboxylative Alkynylation of Aliphatic Carboxylic Acids in Aqueous Solution. J. Am. Chem. Soc. 2012, 134, 14330-14333. [CrossRef] [PubMed]

14. Barker, T.J.; Boger, D.L. Fe(III)/ $\mathrm{NaBH}_{4}$-Mediated Free Radical Hydrofluorination of Unactivated Alkenes. J. Am. Chem. Soc. 2012, 134, 13588-13591. [CrossRef] [PubMed]

15. Li, Z.D.; Song, L.Y.; Li, C.Z. Silver-Catalyzed Radical Aminofluorination of Unactivated Alkenes in Aqueous Media. J. Am. Chem. Soc. 2013, 135, 4640-4643. [CrossRef] [PubMed] 
16. Fujita, M.; Nagano, T.; Schneider, U.; Hamada, T.; Ogawa, C.; Kobayashi, S. Zn-Catalyzed Asymmetric Allylation for the Synthesis of Optically Active Allylglycine Derivatives. Regio- and Stereoselective Formal $\alpha$-Addition of Allylboronates to Hydrazono Esters. J. Am. Chem. Soc. 2008, 130, 2914-2915. [CrossRef] [PubMed]

17. Gordillo, A.; Ortuño, M.A.; Mardomingo, C.L.; Lledós, A.; Ujaque, G.; Jesús, E. Mechanistic Studies on the Pd-Catalyzed Vinylation of Aryl Halides with Vinylalkoxysilanes in Water: The Effect of the Solvent and NaOH Promoter. J. Am. Chem. Soc. 2013, 135, 13749-13763. [CrossRef] [PubMed]

18. Felpin, F.-X.; Landais, Y. Practical Pd/C-Mediated Allylic Substitution in Water. J. Org. Chem. 2005, 70, 6441-6446. [CrossRef] [PubMed]

19. Candeias, N.R.; Gois, P.M.P.; Afonso, C.A.M. Rh(II)-Catalyzed Intramolecular C-H Insertion of Diazo Substrates in Water: Scope and Limitations. J. Org. Chem. 2006, 71, 5489-5497. [CrossRef] [PubMed]

20. Sotto, N.; Billamboz, M.; Carole, C.-V.; Len, C. Selective Pinacol Coupling on Regeneratable Supported Acids in Sole Water. J. Org. Chem. 2015, 80, 6375-6380. [CrossRef] [PubMed]

21. Liu, L.F.; Dong, Y.; Pang, B.; Ma, J.H. [Bmim]PF - -Promoted Ligandless Suzuki-Miyaura Coupling Reaction of Potassium Aryltrifluoroborates in Water. J. Org. Chem. 2014, 79, 7193-7198. [CrossRef] [PubMed]

22. Botella, L.; Nájera, C. Mono- and $\beta, \beta$-Double-Heck Reactions of $\alpha, \beta$-Unsaturated Carbonyl Compounds in Aqueous Media. J. Org. Chem. 2005, 70, 4360-4369. [CrossRef] [PubMed]

23. Erb, W.; Albini, M.; Rouden, J.; Blanchet, J. Sequential One-Pot Access to Molecular Diversity through Aniline Aqueous Borylation. J. Org. Chem. 2014, 79, 10568-10580. [CrossRef] [PubMed]

24. Kalutharage, N.; Yi, C.S. Chemoselective Formation of Unsymmetrically Substituted Ethers from Catalytic Reductive Coupling of Aldehydes and Ketones with Alcohols in Aqueous Solution. Org. Lett. 2015, 17, 1778-1781. [CrossRef] [PubMed]

25. Hikawa, H.; Suzuki, H.; Azumaya, I. Au(III)/TPPMS-Catalyzed Benzylation of Indoles with Benzylic Alcohols in Water. J. Org. Chem. 2013, 78, 12128-12135. [CrossRef] [PubMed]

26. Handa, S.; Slack, E.D.; Lipshutz, B.H. Nanonickel-Catalyzed Suzuki-Miyaura Cross-Couplings in Water. Angew. Chem. Int. Ed. 2015, 54, 11994-11998. [CrossRef] [PubMed]

27. Nishikata, T.; Lipshutz, B.H. AllylicEthersas Educts for Suzuki-Miyaura Couplings in Water at Room Temperature. J. Am. Chem. Soc. 2009, 131, 12103-12105. [CrossRef] [PubMed]

28. Han, J.; Liu, Y.; Guo, R. Facile Synthesis of Highly Stable Gold Nanoparticles and Their Unexpected Excellent Catalytic Activity for Suzuki-Miyaura Cross-Coupling Reaction in Water. J. Am. Chem. Soc. 2009, 131, 2060-2061. [CrossRef] [PubMed]

29. Wei, C.-H.; Mannathan, S.; Cheng, C.-H. Enantioselective Synthesis of $\beta$-Substituted Cyclic Ketones via Cobalt-Catalyzed Asymmetric Reductive Coupling of Alkynes with Alkenes. J. Am. Chem. Soc. 2011, 133, 6942-6944. [CrossRef] [PubMed]

30. Chang, H.-T.; Jayanth, T.T.; Wang, C.-C.; Cheng, C.-H. Cobalt-Catalyzed Reductive Coupling of Activated Alkenes with Alkynes. J. Am. Chem. Soc. 2007, 129, 12032-12041. [CrossRef] [PubMed]

31. Zheng, L.; Zhou, Z.-Z.; He, Y.-T.; Li, L.-H.; Ma, J.-W.; Qiu, Y.-F.; Zhou, P.-X.; Liu, X.-Y.; Xu, P.-F.; Liang, Y.-M. Iodine-Promoted Radical Cyclization in Water: A Selective Reaction of 1,6-Enynes with Sulfonyl Hydrazides. J. Org. Chem. 2016, 81, 66-76. [CrossRef] [PubMed]

32. Xia, X.-F.; Zhu, S.-L.; Chen, C.; Wang, H.J.; Liang, Y.-M. Silver-Catalyzed Decarboxylative Addition/Cyclization of Activated Alkenes with Aliphatic Carboxylic Acids. J. Org. Chem. 2016, 81, 1277-1284. [CrossRef] [PubMed]

33. Cao, J.-L.; Shen, S.-L.; Yang, P.; Qu, J. A Catalyst-Free One-Pot Construction of Skeletons of 5-Methoxyseselin and Alloxanthoxyletin in Water. Org. Lett. 2013, 15, 3856-3859. [CrossRef] [PubMed]

34. Yu, W.S.; Bulger, P.G.; Maloney, K.M. A High-Yielding Method for the Preparation of Isoxazolopyridin3-amine Derivatives. Green Chem. 2016, 18, 4941-4946. [CrossRef]

35. Mase, N.; Nakai, Y.; Ohara, N.; Yoda, H.; Takabe, K.; Tanaka, F.; Barbas, C.F., III. Organocatalytic Direct Asymmetric Aldol Reactions in Water. J. Am. Chem. Soc. 2006, 128, 734-735. [CrossRef] [PubMed]

36. Lerebours, R.; Wolf, C. Palladium(II)-Catalyzed Conjugate Addition of Arylsiloxanes in Water. Org. Lett. 2007, 9, 2737-2740. [CrossRef] [PubMed]

37. Wu, X.-L.; Wang, G.-W. Aminochlorination in Water: First Brønsted Acid-Promoted Synthesis of Vicinal Chloramines. J. Org. Chem. 2007, 72, 9398-9401. [CrossRef] [PubMed] 
38. Zheng, Z.L.; Perkins, B.L.; Ni, B. Diarylprolinol Silyl Ether Salts as New, Efficient, Water-Soluble, and Recyclable Organocatalysts for the Asymmetric Michael Addition on Water. J. Am. Chem. Soc. 2010, 132, 50-51. [CrossRef] [PubMed]

39. He, R.J.; Shirakawa, S.; Maruoka, K. Enantioselective Base-Free Phase-Transfer Reaction in Water-Rich Solvent. J. Am. Chem. Soc. 2009, 131, 16620-16621. [CrossRef] [PubMed]

40. Murase, T.; Nishijima, Y.; Fujita, M. Cage-Catalyzed Knoevenagel Condensation under Neutral Conditions in Water. J. Am. Chem. Soc. 2012, 134, 162-164. [CrossRef] [PubMed]

41. Li, B.; Li, C.B. Neighboring Heteroatom Effect Unique to Aqueous Aldol Reactions of Water-Insoluble Substrates. J. Org. Chem. 2014, 79, 2242-2254. [CrossRef] [PubMed]

42. Zhang, X.; Li, J.; Tian, H.; Shi, Y. Catalytic Asymmetric Bromination of UnfunctionalizedOlefins with $\mathrm{H}_{2}$ Oasa Nucleophile. Chem. Eur. J. 2015, 21, 11658-11663. [CrossRef] [PubMed]

43. Kang, Q.-K.; Wang, L.-J.; Liu, Q.-J.; Li, J.-F.; Tang, Y. Asymmetric $\mathrm{H}_{2} \mathrm{O}-\mathrm{Nucleophilic} \mathrm{Ring} \mathrm{Opening} \mathrm{of} \mathrm{D-A}$ Cyclopropanes: Catalyst Serves as a Source of Water. J. Am. Chem. Soc. 2015, 137, 14594-14597. [CrossRef] [PubMed]

44. Mohan, D.C.; Rao, S.N.; Adimurthy, S. Synthesis of Imidazo[1,2-a]pyridines: "Water-Mediated" Hydroamination and Silver-Catalyzed Aminooxygenation. J. Org. Chem. 2013, 78, 1266-1272. [CrossRef] [PubMed]

45. Hu, P.; Yehoshoa, B.-D.; Milstein, D. General Synthesis of Amino Acid Salts from Amino Alcohols and Basic Water Liberating H . J. Am. Chem. Soc. 2016, 138, 6143-6146. [CrossRef] [PubMed]

46. Miura, T.; Biyajima, T.; Fujii, T.; Murakami, M. Synthesis of $\alpha$-Amino Ketones from Terminal Alkynes via Rhodium Catalyzed Denitrogenative Hydration of N-Sulfonyl-1,2,3-triazoles. J. Am. Chem. Soc. 2012, 134, 194-196. [CrossRef] [PubMed]

47. Buyck, T.; Wang, Q.; Zhu, J.P. Triple Role of Phenylselenonyl Group Enabled a One-Pot Synthesis of 1,3-Oxazinan-2-ones From $\alpha$-Isocyanoacetates, Phenyl Vinyl Selenones, and Water. J. Am. Chem. Soc. 2014, 136, 11524-11528. [CrossRef] [PubMed]

48. Colemana, M.T.D.; Garson, M.J. Marine polypropionates. Nat. Prod. Rep. 1998, 15, 477-493. [CrossRef]

49. Sakakura, A.; Watanabe, H.; Ishihara, K. Rate-Accelerating Effect by the Neighboring-Group Participation of Protecting Groups in the Dehydrative Cyclization of 1,3,5-Triketones. Org. Lett. 2008, 10, 2569-2572. [CrossRef] [PubMed]

50. Boukouvalas, J.; Wang, J.-X. Structure Revision and Synthesis of a Novel Labdane Diterpenoid from Zingiber ottensii. Org. Lett. 2008, 10, 3397-3399. [CrossRef] [PubMed]

51. Molenda, J.J.; Jones, M.M.; Johnston, D.S.; Walker, E.M.; Cannon, D.J. Mobilization of Iron by Chiral and Achiral Anionic 3-Hydroxypyrid-4-one. J. Med. Chem. 1994, 37, 4363-4370. [CrossRef] [PubMed]

52. Stossel, D.; Chan, T.H. A 5C + 5C Bicycloaromatization Reaction via an Aldol Condensation Acyclic Precursors Cascade: A Regioselective Synthesis of Functionalized Naphthalenes from Acyclic Precursors. J. Org. Chem. 1988, 53, 4901-4908. [CrossRef]

53. Reddy, D.S.; Velde, D.V.; Aube, J. Synthesis and Conformational Studies of Dipeptides Constrained by Disubstituted 3-(Aminoethoxy)propionic Acid Linkers. J. Org. Chem. 2004, 69, 1716-1719. [CrossRef] [PubMed]

54. Ma, Y.M.; Luo, W.; Quinn, P.J.; Liu, Z.D.; Hider, R.C. Design, Synthesis, Physicochemical Properties, and Evaluation of Novel Iron Chelators with Fluorescent Sensors. J. Med. Chem. 2004, 47, 6349-6362. [CrossRef] [PubMed]

55. Luo, S.Z.; Mi, X.L.; Xu, H.; Wang, P.G.; Cheng, J.-P. Efficient Baylis-Hillman Reactions of Cyclic Enones in Methanol As Catalyzed by Methoxide Anion. J. Org. Chem. 2004, 69, 8413-8422. [CrossRef] [PubMed]

56. Yeates, C.L.; Batchelor, J.F.; Capon, E.C.; Cheesman, N.J.; Fry, M.; Hudson, A.T.; Pudney, M.; Trimming, H.; Woolven, J.; Bueno, J.M.; et al. Synthesis and Structure-Activity Relationships of 4-Pyridones as Potential Antimalarials. J. Med. Chem. 2008, 51, 2845-2852. [CrossRef] [PubMed]

57. Fakih, S.; Podinovskaia, M.; Kong, X.L.; Collins, H.L.; Schaible, U.E.; Hider, R.C. Targeting the Lysosome: Fluorescent Iron(III) Chelators To Selectively Monitor Endosomal/Lysosomal Labile Iron Pools. J. Med. Chem. 2008, 51, 4539-4552. [CrossRef] [PubMed]

58. Fabiola, B.-J.; Ward, D.E. On the Origin of Siphonariid Polypropionates: Total Synthesis of Caloundrin B and Its Isomerization to Siphonarin B. Org. Lett. 2012, 14, 1648-1651. 
59. Li, D.-F.; Hu, P.-P.; Liu, M.-S.; Kong, X.-L.; Zhang, J.-C.; Hider, R.C.; Zhou, T. Design and Synthesis of Hydroxypyridinone-L-phenylalanine Conjugates as Potential Tyrosinase Inhibitors. J. Agric. Food Chem. 2013, 61, 6597-6603. [CrossRef] [PubMed]

60. Garey, D.; Ramirez, M.-L.; Gonzales, S.; Wertsching, A.; Tith, S.; Keefe, K.; Peña, M.R. An Approach to Substituted 4-Hydroxypyran-2-ones: The Total Synthesis of Phenoxan. J. Org. Chem. 1996, 61, 4853-4856. [CrossRef] [PubMed]

61. Ishibashi, Y.; Ohba, S.; Nishiyama, S.; Yamamura, S. Total Synthesis of Phenoxan and a Related Pyrone Derivative. Tetrahedron Lett. 1996, 37, 2997-3000. [CrossRef]

62. Ehrlich, M.; Carell, T. Total Syntheses and Biological Evaluation of 3-O-Methylfunicone and Its Derivatives Prepared by TMPZnCl-LiCl-Mediated Halogenation and Carbonylative Stille Cross-Coupling. Eur. J. Org. Chem. 2013, 1, 77-83. [CrossRef]

63. Light, R.J.; Hauser, C.R. Aroylations of $p$-Diketones at the Terminal Methyl Group to Form 1,3,5-Triketones. Cyclizations to 4-Pyrones and 4-Pyridones. J. Org. Chem. 1960, 25, 538-546. [CrossRef]

64. Morris, J.; Luke, G.P.; Wishka, D.G. Reaction of Phosgeniminium Salts with Enolates Derived from Lewis Acid Complexes of 2'-Hydroxypropiophenones and Related $\beta$-Diketones. J. Org. Chem. 1996, 61, 3218-3220. [CrossRef] [PubMed]

65. Bunescu, A.; Reimann, S.; Lubbe, M.; Spannenberg, A.; Langer, P. Synthesis of Trifluoromethyl-Substituted Arenes, Cyclohexenones and Pyran-4-ones by Cyclocondensation of 1,3-Bis(silyloxy)-1,3-butadienes with 4,4-Dimethoxy-1,1,1-trifluorobut-3-en-2-one: Influence of the Lewis Acid on the Product Distribution. J. Org. Chem. 2009, 74, 5002-5010. [CrossRef] [PubMed]

66. Malamasa, M.S.; Barnes, K.; Johnson, M.; Hui, Y.; Zhou, P.; Turner, J.; Hu, Y.; Wagner, E.; Fan, K.; Chopra, R.; et al. Di-substituted pyridinyl aminohydantoins as potent and highly selective human $\beta$-secretase (BACE1) inhibitors. Bioorg. Med. Chem. 2010, 18, 630-639. [CrossRef] [PubMed]

67. Weber, F.; Brückner, R. Total Syntheses of the Dihydrofuranonecarboxylate Natural Products Gregatin B and E: Gram-Scale Synthesis of (+)-Gregatin B and Unambiguous Assignment of the Stereostructure of (+)-Gregatin E. Org. Lett. 2014, 16, 6428-6431. [CrossRef] [PubMed]

68. Li, C.-S.; Lacasse, E. Synthesis of Pyran-4-ones from Isoxazoles. Tetrahedron Lett. 2002, 43, 3565-3568. [CrossRef]

69. Jo, Y.-J.; Cho, I.H.; Song, C.K.; Shin, H.W.; Kim, Y.-S. Comparison of Fermented Soybean Paste (Doenjang) Prepared by Different Methods Based on Profiling of Volatile Compounds. J. Food Sci. 2011, 76, C368-C379. [CrossRef] [PubMed]

70. Teng, Q.-H.; Xu, Y.-L.; Liang, Y.; Wang, H.-S.; Wang, Y.-C.; Pan, Y.-M. Transition Metal-Free Synthesis of 3-Alkynylpyrrole-2-carboxylates via Michael Addition/Intramolecular Cyclodehydration. Adv. Synth. Catal. 2016, 358, 1897-1902. [CrossRef]

71. Tan, X.-C.; Liang, Y.; Bao, F.-P.; Wang, H.-S.; Pan, Y.-M. Silver-Mediated C-H Bond Functionalization: One-Pot to Construct Substituted Indolizines from 2-Alkylazaarenes with Alkynes. Tetrahedron 2014, 70, 6717-6722. [CrossRef]

72. Wang, X.; Li, S.-Y.; Pan, Y.-M.; Wang, H.-S.; Liang, H.; Chen, Z.-F.; Qin, X.-H. Samarium(III)-Catalyzed C $\left(\mathrm{sp}^{3}\right)-\mathrm{H}$ Bond Activation: Synthesis of Indolizines via $\mathrm{C}-\mathrm{C}$ and $\mathrm{C}-\mathrm{N}$ Coupling between 2-Alkylazaarenes and Propargylic Alcohols. Org. Lett. 2014, 16, 580-583. [CrossRef] [PubMed]

73. Liu, P.; Pan, Y.-M.; Xu, Y.-L.; Wang, H.-S. PTSA-Catalyzed Mannich-Type-Cyclization-Oxidation Tandem Reactions: One-Pot Synthesis of 1, 3, 5-Substituted Pyrazoles from Aldehydes, Hydrazines and Alkynes. Org. Biomol. Chem. 2012, 10, 4696-4698. [CrossRef] [PubMed]

74. Wang, X.; Pan, Y.-M.; Huang, X.-C.; Mao, Z.-Y.; Wang, H.-S. A novel methodology for synthesis of dihydropyrazole derivatives as potential anticancer agents. Org. Biomol. Chem. 2014, 12, 2028-2032. [CrossRef] [PubMed]

75. Pan, Y.-M.; Zheng, F.-J.; Lin, H.-X.; Zhan, Z.-P. Brønsted Acid-Catalyzed Propargylation/Cycloisomerization Tandem Reaction: One-Pot Synthesis of Substituted Oxazoles from Propargylic Alcohols and Amides. J. Org. Chem. 2009, 74, 3148-3151. [CrossRef] [PubMed]

76. Xie, H.-Z.; Gao, Q.; Liang, Y.; Wang, H.-S.; Pan, Y.-M. Palladium-Catalyzed Synthesis of Benzoxazoles by the Cleavage Reaction of Carbon-Carbon Triple Bonds with o-Aminophenol. Green Chem. 2014, 16, 2132-2135. [CrossRef] 
77. Wang, Y.-C.; Wang, H.-S.; Huang, G.-B.; Huang, F.-P.; Hu, K.; Pan, Y.-M. A One-Pot Approach to 4,5-Dihydropyrazoles from Ketones, Arylacetylenes, and Hydrazines. Tetrahedron 2014, 70, 1621-1628. [CrossRef]

78. Qiu, Y.-F.; Yang, F.; Qiu, Z.-H.; Zhong, M.-J.; Wang, L.-J.; Ye, Y.-Y.; Song, B.; Liang, Y.-M. Brønsted Acid Catalyzed and NIS-Promoted Cyclization of Diynones: Selective Synthesis of 4-Pyrone, 4-Pyridone, and 3-Pyrrolone Derivatives. J. Org. Chem. 2013, 78, 12018-12028. [CrossRef] [PubMed]

79. Yang, F.; Qiu, Y.-F.; Ji, K.-G.; Niu, Y.-N.; Ali, S.; Liang, Y.-M. Divergent Synthesis of Benzene Derivatives: Brønsted Acid Catalyzed and Iodine-Promoted Tandem Cyclization of 5,2-Enyn-1-ones. J. Org. Chem. 2012, 77, 9029-9037. [CrossRef] [PubMed]

80. Yang, F.; Ji, K.-G.; Zhao, S.-C.; Ali, S.; Ye, Y.-Y.; Liu, X.-Y.; Liang, Y.-M. Brønsted Acid Catalyzed Cycloisomerizations of 5,2-Enyne-1-ones: Highly Regioselective Synthesis of 2,3-Dihydro-4H-pyran-4-ones. Chem. Eur. J. 2012, 18, 6470-6474. [CrossRef] [PubMed]

81. Morisaki, Y.; Luu, T.; Tykwinski, R.R. A One-Pot Synthesis and Functionalization of Polyynes. Org. Lett. 2006, 8, 689-692. [CrossRef] [PubMed]

82. Knight, J.D.; Metz, C.R.; Beam, C.F.; Pennington, W.T.; Derveer, D.G.V. New Strong Base Synthesis of Symmetrical 1,5-Diaryl-1,3,5-pentanetriones from Acetone and Benzoate Esters. Synth. Commun. 2008, 38, 2465-2482. [CrossRef]

83. Aziz, S.; Mahnaz, S. Synthesis of Pyrone Carbaldehydes, Pyrone Sulfonium Ylides and Related Epoxides. J. Heterocycl. Chem. 2009, 46, 268-272.

84. Jobour, A.; Nazar, H.; Shandala, M.Y. Synthesis and Spectral Data of Some Heterocyclic Compounds. The Reaction of Arylpropiolic Esters with Tetralones and Acetylcyclopropane. J. Heterocycl. Chem. 1980, 17, 941-944. [CrossRef]

85. Toshiaki, S.; Taichi, O.; Kiyomi, S.; Yosihiro, N.; Mitsuru, H.; Yoshie, H.; Jun, T.; Takehiro, S. Thermal Addition Reaction of Aroylketene with 1-Aryl-1-trimethylsilyloxyethylenes: Aromatic Substituent Effects of Aroylketene and Aryltrimethylsilyloxyethylene on Their Reactivity. Chem. Pharm. Bull. 1996, 44, 956-966.

86. Groundwater, P.W.; Hibbs, D.E.; Hursthousea, M.B.; Nyerges, M. Synthesis and Reactions of Reduced Flavones. J. Chem. Soc. Perkin Trans. 1 1997, 163-170. [CrossRef]

Sample Availability: Samples of the compounds $\mathbf{2 a}-\mathbf{2 r}, \mathbf{2 a -} \boldsymbol{d}$ and $\mathbf{2 a} \mathbf{a}-\mathbf{o}^{\mathbf{1 8}}$ are available from the authors.

(c) 2017 by the authors; licensee MDPI, Basel, Switzerland. This article is an open access article distributed under the terms and conditions of the Creative Commons Attribution (CC-BY) license (http://creativecommons.org/licenses/by/4.0/). 